\title{
Association between new anthropometric parameters and arterial stiffness based on brachial-ankle pulse wave velocity
}

This article was published in the following Dove Press journal: Diabetes, Metabolic Syndrome and Obesity: Targets and Therapy

\author{
Hee Seon Choi ${ }^{1}$ \\ Young Hye Cho' \\ Sang Yeoup Lee \\ Eun Ju Park' \\ Yun Jin $\mathrm{Kim}^{2,3}$ \\ Jeong Gyu Lee ${ }^{2,3}$ \\ Yu Hyeon $\mathrm{Yi}^{2,3}$ \\ Young Jin $\mathrm{Tak}^{2,3}$ \\ Hye Rim Hwang ${ }^{2,3}$ \\ Seung Hun Lee 2,3 \\ 'Family Medicine Clinic and Research \\ Institute of Convergence of Biomedical \\ Science and Technology, Pusan Nationa \\ University Yangsan Hospital, Yangsan, \\ Gyeongsangnam-do 506/2, South Korea; \\ ${ }^{2}$ Department of Family Medicine, Pusan \\ National University School of Medicine, \\ Yangsan 626-780, South Korea; \\ ${ }^{3}$ Department of Family Medicine and \\ Biomedical Research Institute, Pusan \\ National University Hospital, Busan \\ 626-770, South Korea
}

Background: Obesity is an important risk factor of cardiovascular disease (CVD). Atherosclerosis can be considered an important signal of CVD. Primary physicians can reduce the risk of CVD by preventing and treating obesity. Therefore, finding a tool to diagnose the association of obesity with arteriosclerosis is important. The association between obesity parameters and arterial stiffness remains controversial. To our knowledge, no previous studies reported the relationships between multiple new anthropometric parameters (a body shape index [ABSI], body round index [BRI], and visceral adiposity index [VAI]) and brachial-ankle wave velocity (ba-PWV) as an indicator of CVD risk, especially in the Korean population.

Objective: To investigate the relationships between arterial stiffness (assessed using baPWV) and anthropometric parameters estimated on the basis of body mass index (BMI), waist circumference (WC), ABSI, BRI, and VAI, and to identify the indicators of obesity that best represents CVD risk.

Methods: A total of 2,647 adults (1,474 men and 1,173 women) were recruited for this cross-sectional study. The correlations between the anthropometric indexes (BMI, WC, ABSI, BRI, and VAI) and mean ba-PWV were analyzed. A multivariate linear regression analysis was performed to evaluate the association between each anthropometric and the presence of arterial stiffness.

Results: We investigated the relationships between the obesity parameters and ba-PWV by adjusting the covariates (age, diabetes mellitus [DM], hypertension [HTN], and smoking status) related to the mean ba-PWV. In the multivariate regression analysis, ABSI (men: $\beta=0.066, p<0.01$; women: $\beta=0.087, p<0.001$ ) and VAI (men: $\beta=0.067, p<0.01$; women: $\beta=0.136, p<0.001$ ) were found to be significantly correlated with the mean ba-PWV in both men and women in Korea.

Conclusion: Among the new obesity indices, ABSI and VAI were found to be significantly associated with arterial stiffness, represented by the mean ba-PWV, in both men and women in Korea. These results suggest that ABSI and VAI may be convenient, highly cost-effective, and simple assessment tools for obesity and CVD risk in clinical practice.

Keywords: anthropometric index, arterial stiffness, brachial-ankle pulse wave velocity

\section{Introduction}

Obesity is defined as a physiological state of chronic imbalance in systemic energy metabolism in which the caloric intake exceeds energy expenditure. ${ }^{1}$ Visceral fat can especially produce many compounds with autocrine, paracrine, and endocrine activities, which could influence metabolism and the cardiovascular system. ${ }^{2}$
Correspondence: Young Hye Cho Metabolism and Nutrition Center and Biomedical Science and Technology, Pusan National University Yangsan Hospital, Yangsan 626-770, South Korea

Tel +82 5I 2407707

Fax +82512407843

Email younghey82@naver.com 
Therefore, obesity is an important risk factor of cardiovascular disease (CVD). ${ }^{2-4}$ Physicians can play an important role in reducing the CVD risk through obesity prevention and treatment. Therefore, identifying a tool to diagnose obesity, which has been found to be significantly associated with arteriosclerosis, that can be easily used in clinical practice is important. The body mass index (BMI) has been widely used as a diagnostic index of general obesity, but it does not differentiate muscle and fat masses, or reflect fat distribution. ${ }^{2,5}$ Waist circumference (WC), a simple anthropometric parameter of abdominal obesity or fat distribution, has been suggested as an alternative to BMI. However, several studies have revealed that $\mathrm{WC}$ is highly correlated to BMI; consequently, its usefulness on its own is limited. ${ }^{6,7}$ To overcome these limitations, new anthropometric parameters were identified. In 2010, Amato et al developed the visceral adiposity index (VAI), a sex-specific indicator of visceral fat distribution based on WC and BMI, and triglyceride (TG) and high-density lipoprotein-cholesterol (HDL-C). ${ }^{8-10}$ In the study of Amato and Giordano, ${ }^{10}$ the VAI was discovered as a valuable index of visceral fat function and insulin sensitivity, which showed high correlation with cardiometabolic risk. Krakauer et al proposed a new indicator known as a body shape index (ABSI), using a standardized WC based on BMI and height, and found that it reflects mortality hazard better than BMI and WC. ${ }^{2,11,12}$ The body roundness index (BRI), an indicator of body fat and visceral adipose tissue percentage, was invented by Thomas et al (2013) and is suggested to be useful for evaluating health status. ${ }^{2,13}$

Arterial stiffness is a pathological disorder that contributes to both CVD and mortality due to vascular damage. Aortic pulse wave velocity (aPWV) is an independent predictor of CVD. ${ }^{14}$ A recent study by Tsuchikura and Shoji ${ }^{15}$ showed that brachial-ankle wave velocity (ba-PWV), a more convenient tool to evaluate arterial stiffness, is highly correlated with aPWV. Therefore, ba-PWV can also be used as a surrogate marker of CVD risk. ${ }^{9,15}$

Previous studies showed that obesity parameters were risk factors of arterial stiffness, but this finding has been controversial. To our knowledge, no previous studies reported the relationship between multiple new anthropometric parameters (ABSI, BRI, and VAI) and ba-PWV, as an indicator of CVD risk, especially in the Korean population. Therefore, this study aimed to investigate the relationship between arterial stiffness (assessed using
ba-PWV) and anthropometric parameters estimated on the basis of BMI, WC, ABSI, BRI, and VAI.

\section{Methods}

\section{Participants}

We conducted a cross-sectional study based on data obtained from medical records. We included participants who underwent a routine health checkup at a health promotion center of the Pusan National University Hospital between January 2013 and December 2015. A total of 398 individuals were excluded on the basis of the following criteria: ABI $<0.9$, implying the presence of peripheral arterial disease; BMI $<18.5 \mathrm{~kg} / \mathrm{m}^{2}$; history of CVD (history of coronary artery disease, myocardial infarction, stroke, and history of medication for diagnosed angina); and ba-PWV $>3.0$ standard deviations from the mean value. Finally, 2,647 adults (1,474 men and 1,173 women) were included in this study.

All the participants completed a questionnaire and were interviewed by the physician regarding their medical history, family history, and lifestyle information (including smoking status, alcohol consumption, and exercise habit). Diabetes mellitus (DM), hypertension (HTN), or dyslipidemia history was defined as a history of medication use or physician diagnosis. CVD history was defined as the presence of a previous coronary artery disease, myocardial infarction, stroke, and history of medication use for a diagnosed angina. Smoking status was classified as smoker (current and ex-smokers) and non-smoker (those who had never smoked). Moderate drinking was defined as consuming $>14 \mathrm{~g}$ of alcohol per day for women and $>28 \mathrm{~g}$ of alcohol per day for men. ${ }^{16}$ Physical activity was defined as moderate to severe physical activity for $>150 \mathrm{~min}$ per week. $^{17}$

This study was eligible for exemption from IRB review because it was based on retrospective medical record and the data was anonymous. The study protocol was approved by the Institutional Review Board of Pusan National University Hospital (PNUH, IRB No. H-1811-004-072).

\section{Data collection}

\section{Anthropometric parameters}

Weight and height were measured in light clothing and without wearing shoes by using a digital scale with an accuracy of $0.1 \mathrm{~kg}$ and $0.1 \mathrm{~cm}$, respectively. WC was measured with a tape measure in the middle of the lower border of the ribs and the iliac crest. BMI was 
calculated as weight $(\mathrm{kg})$ divided by the square of height (m).

ABSI, BRI, and VAI were calculated using the following formulas: ${ }^{9,18}$

- ABSI

$$
\mathrm{ABSI}=\frac{\mathrm{WC}}{\left(\mathrm{BMI}^{\frac{2}{3}} \times \text { height }^{\frac{1}{2}}\right)}
$$

- BRI

$$
\mathrm{BRI}=364.2-365 \cdot 5 \times \sqrt{1-\frac{\left(\frac{\mathrm{WC}}{2 \pi}\right)^{2}}{(0.5 \times \text { height })^{2}}}
$$

- VAI

$$
\begin{aligned}
\text { Males : VAI } & =\left(\frac{\mathrm{WC}}{39.68+(1.88 \times \mathrm{BMI})}\right) \times\left(\frac{\mathrm{TG}}{1.03}\right) \\
& \times\left(\frac{1.31}{\mathrm{HDL}}\right) \\
\text { Females : VAI } & =\left(\frac{\mathrm{WC}}{36.58+(1.89 \times \mathrm{BMI})}\right) \times\left(\frac{\mathrm{TG}}{0.81}\right) \\
& \times\left(\frac{1.52}{\mathrm{HDL}}\right)
\end{aligned}
$$

\section{Ba-pwv measurement}

Ba-PWV as an indicator of arterial stiffness ${ }^{9}$ was measured in supine position after a 5-min rest by using an automated device (Model BP-203RPE-III, Omron, Japan). The instrument collected waveform data from cuff sensors applied on the right brachium and both ankles. The ba-PWV value was automatically calculated using the following equation: ba$\mathrm{PWV}=(\mathrm{La}-\mathrm{Lb}) / \mathrm{Tba}$. Tba represents that time intervals between the brachium and the ankle. La represents the distance from the heart to the ankle in the following equation: $(\mathrm{La})=0.8129 \times$ height $(\mathrm{cm})+12.328$. Lb represents the distance from the heart to the right brachium in the following equation: $(\mathrm{Lb})=0.2195 \times$ height $(\mathrm{cm})-2.0734 .{ }^{19}$ The mean ba-PWV (right and left) values were used for the analysis.

\section{Laboratory measurements}

Venous blood samples were obtained from the antecubital vein after an overnight fast of at least $8 \mathrm{~h}$ to measure the total cholesterol, HDL-C, TG, and fasting plasma glucose levels using a certified auto-analyzer (Hitachi 7600 Analyzer [Hitachi Co., Ltd., Tokyo, Japan] and Synchron LX 20 [Beckman Coulter, Fullerton, CA, USA]). Highsensitivity C-reactive protein (hs-CRP) levels were assessed using a Behring BN II Nephelometer (Dade Behring, Marburg, Germany).

\section{Statistical analyses}

The baseline characteristics of continuous data were presented as mean and standard deviation, whereas categorical data were expressed as frequencies (n, number) and percentages (\%). The chi-square or two-sample $t$-test was used to compare demographic and clinical characteristics between the men and women. The Pearson correlation coefficients are calculated to determine the correlation between the anthropometric parameters and the mean baPWV. Considering the difference between the anthropometric parameters and the mean ba-PWV in the men and women, the participants were divided into two groups according to sex, and the data were analyzed separately. A standardized linear regression analysis was performed to determine the effects of demographic and clinical characteristics, and anthropometric parameters on the mean baPWV, unadjusted and adjusted for age. To examine the association between the obesity parameters and the mean ba-PWV, and the covariates, a final multivariate linear regression analysis was performed. A two-tailed $p$-value of $<0.05$ was considered statistically significant. All statistical analyses were performed using SPSS ver. 21.0 for Windows (SPSS, Inc., Chicago, Illinois, USA).

\section{Results}

The baseline characteristics of 2,467 participants are presented in Table 1. The participants consisted of 1,474 men $(55.69 \%)$ and 1,173 women $(44.31 \%)$, with a mean age of $46.42 \pm 11.74$ years (men: $47.19 \pm 11.22$ years; women: $45.45 \pm 12.30$ years). The mean ba-PWV was 1300.53 \pm 209.61 and was statistically significantly higher in the men than in the women $(1345.30 \pm 187.39$ vs 1244.27 $\pm 222.20, p<0.001)$. Compared with the women, the men were older and exhibited significantly higher values of the anthropometric indexes (BMI, WC, ABSI, BRI, and VAI) and biomarkers such as total cholesterol, TG, HDL, and glucose levels, except CRP level $(p=0.297)$. As the unit of ABSI is too small, ABSI $\times 100$ is represented in Tables 1 , 3 , and 4. No significant differences in diabetes mellitus and dyslipidemia histories and physical activity were 
Table I Baseline characteristics of the participants $N=2647$

\begin{tabular}{|c|c|c|c|c|}
\hline Variable & $\begin{array}{l}\text { Total } \\
(n=2647)\end{array}$ & $\begin{array}{l}\text { Male } \\
(n=1474)\end{array}$ & $\begin{array}{l}\text { Female } \\
(n=I \mid 73)\end{array}$ & $\mathbf{p}^{\ddagger}$ \\
\hline Age $(y)$ & $46.42 \pm I I .74$ & $47.19 \pm 11.22$ & $45.45 \pm 12.30$ & $<0.001 * * *$ \\
\hline \multicolumn{5}{|l|}{ Anthropometric Index } \\
\hline BMI $\left(\mathrm{kg} / \mathrm{m}^{2}\right)$ & $23.83 \pm 3.16$ & $24.65 \pm 2.91$ & $22.79 \pm 3.16$ & $<0.001^{* * *}$ \\
\hline$W C(\mathrm{~cm})$ & $82.97 \pm 9.12$ & $86.67 \pm 7.83$ & $78.31 \pm 8.48$ & $<0.001 * * *$ \\
\hline ABSI $\times 100$ & $7.77 \pm 0.43$ & $7.81 \pm 0.35$ & $7.72 \pm 0.50$ & $<0.001 * * *$ \\
\hline BRI & $3.35 \pm 0.97$ & $3.46 \pm 0.86$ & $3.23 \pm 1.08$ & $<0.001 * * *$ \\
\hline VAl & $1.45 \pm 1.4 \mathrm{I}$ & $1.61 \pm 1.43$ & $1.24 \pm 1.36$ & $<0.001 * * *$ \\
\hline \multicolumn{5}{|l|}{ Biochemical Markers } \\
\hline $\mathrm{TC}(\mathrm{mg} / \mathrm{dL})$ & $190.95 \pm 36.30$ & $194.03 \pm 36.03$ & $187.28 \pm 34.94$ & $<0.001 * * *$ \\
\hline TG (mmol/L) & $1.24 \pm 0.87$ & $1.46 \pm 0.97$ & $0.91 \pm 0.48$ & $<0.001 * * *$ \\
\hline $\mathrm{HDL}(\mathrm{mmol} / \mathrm{L})$ & $1.47 \pm 0.40$ & $1.34 \pm 0.35$ & $1.64 \pm 0.39$ & $<0.001 * * *$ \\
\hline AST & $22.48 \pm|1.9|$ & $24.63 \pm 13.30$ & $19.79 \pm 9.22$ & $<0.001 * * *$ \\
\hline BUN & $|2.74 \pm 4.2|$ & $13.36 \pm 4.48$ & $11.97 \pm 3.70$ & $<0.00 I^{* * *}$ \\
\hline $\mathrm{Cr}$ & $0.80 \pm 0.38$ & $0.92 \pm 0.46$ & $0.65 \pm 0.12$ & $<0.001 * * *$ \\
\hline Glu (mg/dL) & $90.12 \pm 20.11$ & $92.80 \pm 23.15$ & $86.75 \pm|4.8|$ & $<0.00 I^{* * *}$ \\
\hline Uric acid & $5.19 \pm 1.44$ & $5.95 \pm 1.30$ & $4.25 \pm 0.97$ & $<0.001^{* * *}$ \\
\hline CRP & $0.12 \pm 0.36$ & $0.13 \pm 0.31$ & $0.11 \pm 0.42$ & 0.297 \\
\hline Mean ba-PWV & $|300.53 \pm 209.6|$ & $\mid 345.30 \pm 187.39$ & $1244.27 \pm 222.20$ & $<0.001 * * *$ \\
\hline $\mathrm{SBP}(\mathrm{mmHg})$ & $120.82 \pm 15.66$ & $124.66 \pm 14.77$ & $116.00 \pm 15.43$ & $<0.001 * * *$ \\
\hline $\mathrm{DBP}(\mathrm{mmHg})$ & $74.28 \pm 10.76$ & $77.05 \pm 10.44$ & $70.79 \pm 10.12$ & $<0.00 I^{* * *}$ \\
\hline HR (bpm) & $72.95 \pm 10.50$ & $71.84 \pm 10.23$ & $74.35 \pm 10.67$ & $<0.001 * * *$ \\
\hline \multicolumn{5}{|l|}{ Disease History ${ }^{\mathrm{a}}$} \\
\hline Diabetes mellitus, n (\%) & 4.3 & 5.1 & 3.4 & 0.35 \\
\hline Hypertension, n (\%) & 13.1 & 15.9 & 9.5 & $<0.001^{* * *}$ \\
\hline Dyslipidemia, n (\%) & 10.2 & 11.3 & 8.7 & 0.26 \\
\hline \multicolumn{5}{|l|}{ Health-related Behavior } \\
\hline Smoking ${ }^{\mathrm{b}}, \mathrm{n}(\%)^{\mathrm{b}}$ & 43.4 & 72.9 & 6.5 & $<0.001 * * *$ \\
\hline Drinking $^{c}, \mathrm{n}(\%)^{\mathrm{c}}$ & 47.8 & 63.5 & 28.1 & $<0.00 I^{* * *}$ \\
\hline Inactivity $^{d}, \mathrm{n}(\%)^{d}$ & 80.2 & 79.3 & 81.4 & 0.176 \\
\hline
\end{tabular}

Notes: ${ }^{* * *} p<0.00 \mathrm{I}$. Values are presented as mean \pm SD or number (percentage). ${ }^{\ddagger}$ The $p$-value was calculated using $t$ and $\chi^{2}$ tests. ${ }^{\mathrm{a}} \mathrm{Disease}$ history based on medication or the physician's diagnosis. ' Non-smokers, never smoked; smokers, have smoked. 'Moderate drinking ( $\geq 14 \mathrm{~g} /$ day of alcohol for women and $\geq 24 \mathrm{~g} /$ day of alcohol for men). ${ }^{\mathrm{I}}$ Inactivity, did not perform moderate-intensity exercise at least $30 \mathrm{~min}$.

Abbreviations: BMI, Body mass index; WC, Waist circumference; ABSI, A body shape index; BRI, Body round index; VAI, Visceral adiposity index; TC, Total cholesterol; TG, Triglyceride; HDL, High-density lipoprotein; AST, Aspartate aminotransferase; BUN, Blood urea nitrogen; Cr, Creatinine; Glu, Glucose; CRP, C-reactive protein; baPWV, Brachial-ankle pulse wave velocity; SBP, Systolic blood pressure; DBP, Diastolic blood pressure; HR, Heart rate.

observed between the men and women. However, the male participants predominantly consisted of participants with a hypertension history (men: 15.9\%: women; 9.5\%, $p<0.001$ ), smoking status (men: $72.9 \%$; women: $6.5 \%$, $p<0.001$ ), and drinking history (men: 63.5\%; women: $28.1 \%, p<0.001)$. No significant sex-related differences were found in the proportion of participants in the inactive groups who did not have an exercise history (men: $79.3 \%$; women: $81.4 \%, p=0.176$ ).

In this study, traditional CVD risk factors such as systolic blood pressure (SBP), diastolic blood pressure (DBP), heart rate, and levels of total cholesterol, TGs, and HDL were used to evaluate the correlations between the obesity indexes and CVD. The results are shown in Table 2. SBP, DBP, TC, and TG levels positively correlated and HDL level negatively correlated with all the obesity indexes. In addition, the mean ba-PWV, which represents atherosclerosis, was significantly associated with all the anthropometric parameters.

We confirmed whether disease history, social history (including smoking and drinking statuses), and obesity indices are associated with ba-PWV (Table 3). As a result, DM and HTN history and smoking status significantly correlated with mean ba-PWV. In the men, BMI was not 
Table 2 Correlations of the anthropometric parameters with the CVD risk factors

\begin{tabular}{|l|l|l|l|l|l|l|l|l|l|}
\hline & BMI & WC & ABSI & BRI & VAI & AST & Cr & Glu & CRP \\
\hline SBP & $0.384^{* *}$ & $0.391^{* *}$ & $0.116^{* *}$ & $0.356^{* *}$ & $0.183^{* *}$ & $0.170^{* *}$ & $0.186^{* *}$ & $0.173^{* *}$ & $0.040^{*}$ \\
DBP & $0.367^{* *}$ & $0.368^{* *}$ & $0.081^{* *}$ & $0.313^{* *}$ & $0.197^{* *}$ & $0.162^{* *}$ & $0.165^{* *}$ & $0.166^{* *}$ & 0.029 \\
HR & -0.036 & $-0.051^{* *}$ & -0.007 & -0.012 & $0.048^{*}$ & 0.015 & -0.021 & $0.075^{* *}$ & $0.102^{* *}$ \\
TC & $0.196^{* *}$ & $0.195^{* *}$ & $0.085^{* *}$ & $0.207^{* *}$ & $0.240^{* *}$ & $0.169^{* *}$ & $0.046^{*}$ & $0.118^{* *}$ & -0.011 \\
TG & $0.357^{* *}$ & $0.362^{* *}$ & $0.059^{* *}$ & $0.274^{* *}$ & $0.913^{* *}$ & $0.173^{* *}$ & $0.095^{* *}$ & $0.243^{* *}$ & $0.060^{* *}$ \\
HDL & $-0.363^{* *}$ & $-0.379^{* *}$ & $-0.062^{* *}$ & $-0.270^{* *}$ & $-0.522^{* *}$ & $-0.096^{* *}$ & $-0.152^{* *}$ & $-0.139^{* *}$ & $-0.099^{* *}$ \\
Mean ba-PWV & $0.179^{* *}$ & $0.259^{* *}$ & $0.257^{* *}$ & $0.303^{* *}$ & $0.160^{* *}$ & $0.210^{* *}$ & $0.163^{* *}$ & $0.263^{* *}$ & $0.074^{* *}$ \\
\hline
\end{tabular}

Notes: ${ }^{*} p<0.05,{ }^{*} p<0.01$, for the correlation coefficient.

Abbreviations: BMI, Body mass index; WC, Waist circumference; ABSI, A body shape index; BRI, Body round index; VAI, Visceral adiposity index; SBP, Systolic blood pressure; DBP, Diastolic blood pressure; HR, Heart rate; TC, Total cholesterol; TG, Triglycerides; HDL, High-density lipoprotein; ba-PWV, Brachial-ankle pulse wave velocity.

Table 3 Association between the mean ba-PWV and the clinical characteristics or obesity parameters

\begin{tabular}{|c|c|c|c|c|c|c|c|c|c|c|c|c|}
\hline \multirow[t]{3}{*}{ Variable } & \multicolumn{6}{|l|}{ Male } & \multicolumn{6}{|c|}{ Female } \\
\hline & \multicolumn{3}{|c|}{ Unadjusted } & \multicolumn{3}{|c|}{ Adjusted for age } & \multicolumn{3}{|c|}{ Unadjusted } & \multicolumn{3}{|c|}{ Adjusted for age } \\
\hline & $\boldsymbol{\beta}$ & SE & $p$ & $\beta$ & SE & $p$ & $\boldsymbol{\beta}$ & SE & $p$ & $\beta$ & SE & $p$ \\
\hline Age & 0.476 & 0.383 & & & & & 0.690 & 0.382 & $*$ & & & \\
\hline Diabetes mellitus & 0.192 & 21.803 & $*$ & 0.111 & 19.711 & $*$ & 0.155 & 35.331 & $*$ & $0.05 I$ & 26.132 & 0.018 \\
\hline Hypertension & 0.222 & 13.025 & $*$ & 0.098 & 12.170 & $*$ & 0.329 & 20.852 & $*$ & 0.094 & $17.00 \mid$ & $*$ \\
\hline Dyslipidemia & 0.086 & 15.347 & 0.001 & 0.035 & 13.625 & 0.134 & 0.183 & 22.645 & $*$ & 0.029 & 17.098 & 0.175 \\
\hline Smoking & 0.045 & 10.969 & 0.082 & 0.010 & 9.688 & 0.659 & -0.072 & 26.299 & 0.014 & 0.050 & 19.336 & 0.021 \\
\hline Drinking & -0.082 & 10.107 & 0.002 & 0.028 & 9.162 & 0.232 & -0.178 & 14.204 & $*$ & 0.017 & 10.885 & 0.429 \\
\hline Inactivity & -0.010 & 12.052 & 0.702 & 0.042 & 10.657 & 0.067 & -0.036 & 16.675 & 0.219 & 0.030 & 12.122 & 0.162 \\
\hline BMI & 0.012 & 1.679 & 0.656 & 0.009 & 1.477 & 0.684 & 0.220 & 2.006 & $*$ & 0.121 & 1.485 & $*$ \\
\hline WC & 0.053 & 0.623 & 0.044 & 0.022 & 0.549 & 0.335 & 0.292 & 0.732 & $*$ & 0.142 & 0.559 & $*$ \\
\hline ABSI $\times 100$ & 0.198 & 13.517 & $*$ & 0.075 & 12.562 & 0.002 & 0.273 & 12.454 & $*$ & 0.095 & 9.648 & $*$ \\
\hline BRI & 0.162 & 5.586 & $*$ & 0.063 & 5.087 & 0.007 & 0.389 & 5.550 & $*$ & 0.182 & 4.485 & $*$ \\
\hline VAI & 0.045 & 3.413 & 0.083 & 0.072 & 3.001 & 0.002 & 0.235 & 4.633 & $*$ & 0.147 & 3.410 & $*$ \\
\hline
\end{tabular}

Notes: ${ }^{*} p<0.001$, for the correlation coefficient. The $p$-values were obtained using a generalized regression analysis.

Abbreviatiopns: $\beta$, Standardized coefficient; ba-PWV, Brachial-ankle pulse wave velocity; BMI, Body mass index; WC, Waist circumference; ABSI, A body shape index; BRI, Body round index; VAl, Visceral adiposity index.

statistically significantly related to ba-PWV even before age adjustment. After adjusting for age, ABSI, BRI, and VAI were associated with mean ba-PWV. In the men, DM and HTN histories were significantly associated with mean ba-PWV after adjusting for age. In the women, all the anthropometric parameters were positively associated with the mean ba-PWV. DM and HTN histories, and smoking status were significantly associated with the mean ba-PWV after adjusting for age in the women (Table 3).

Finally, the relationships between the obesity parameters and ba-PWV were investigated by adjusting for covariates (men: DM and HTN histories; women: DM history, HTN history, and smoking) related to the mean ba-PWV. In the multivariate regression analysis, ABSI (men: $\beta=0.066, p<0.01$ ) and VAI (men: $\beta=0.067, p<0.01$ ) were found to be associated with the mean ba-PWV in the men. By contrast, all the obesity indices (WC: $\beta=0.129$, $p<0.01$; BMI: $\beta=0.108, p<0.01$; ABSI $\times 100: \beta=0.087$, $p<0.01$; BRI: $\beta=0.167, p<0.01$; and VAI: $\beta=0.136$, $p<0.01)$ were significantly associated with ba-PWV only in the women. In the men, BMI was negatively associated with the mean ba-PWV, and the other parameters such as WC and BRI were not statistically significant.

\section{Discussion}

Obesity is an important risk factor of cardiovascular disease (CVD). Atherosclerosis can be considered an important signal of CVD. Primary physicians can reduce the CVD risk by preventing and treating obesity. Therefore, identifying a tool to diagnose obesity, which is associated with arteriosclerosis, is important. Evaluation of ba-PWV 
Table 4 Association between the mean ba-PWV and the anthropometric parameters

\begin{tabular}{|c|c|c|c|c|c|c|c|c|}
\hline \multirow[t]{2}{*}{ Mean ba-PWV } & \multicolumn{4}{|l|}{ Male } & \multicolumn{4}{|c|}{ Female } \\
\hline & B & $\beta$ & $p$ & $95 \% \mathrm{Cl}$ & B & $\beta$ & $p$ & $95 \% \mathrm{Cl}$ \\
\hline WC & 0.033 & 0.001 & 0.953 & -1.056 to 1.121 & 3.375 & 0.129 & $*$ & $2.272-4.477$ \\
\hline BMI & -0.654 & -0.010 & 0.660 & -3.275 to 2.264 & 7.631 & 0.108 & $*$ & $4.709-10.553$ \\
\hline$A B S I \times 100$ & 34.895 & 0.066 & 0.005 & $10.362-59.429$ & 38.667 & 0.087 & $*$ & 19.799-57.535 \\
\hline BRI & 9.080 & 0.042 & 0.078 & -1.008 to 19.168 & 34.522 & 0.167 & $*$ & $25.6 \mathrm{II}-43.433$ \\
\hline VAI & 8.771 & 0.067 & 0.003 & $2.93 \mathrm{I}-14.6 \mathrm{II}$ & 22.258 & 0.136 & $*$ & $|5.535-28.98|$ \\
\hline
\end{tabular}

Notes: ${ }^{*} p<0.00$ I, for the correlation coefficient. Adjustment for age, hypertension history, diabetes mellitus history, and smoking status. The $p$-values were obtained using a generalized regression analysis.

Abbreviations: $\beta$, Standardized coefficient; ba-PWV, Brachial-ankle pulse wave velocity; Cl, confidence interval; BMI, Body mass index; WC, Waist circumference; ABSI, A body shape index; BRI, Body round index; VAI, Visceral adiposity index.

is crucial in predicting the risk of cardiovascular events among individuals. ${ }^{20}$

We considered the mean ba-PWV as a risk factor for CVD and investigated its association with obesity index (Table 2). In addition, DM and HTN histories, and smoking status were significantly correlated with the mean baPWV (Table 3). Finally, this cross-sectional study found that ABSI and VAI were significantly associated with the mean ba-PWV in both sexes (Table 4).

Unexpectedly, BMI negatively correlated with ba-PWV in the men (Table 4). BMI, WC, and BRI were statistically significantly associated with mean ba-PWV only in the women. This sex-specific difference is based on body fat distribution. Visceral fat tends to be predominant in men, whereas subcutaneous fat is predominant in women; this difference might confirm the existence of such sex-related difference. $^{21}$ Therefore, our findings can be partially explained by the fact that BMI does not differentiate between fat and muscle or does not reflect fat distribution. ${ }^{5}$ On the basis of the study by Czernichow et al, ${ }^{22}$ ba-PWV did not correlate with BMI or body fat in the middle-aged participants. The lack of a significant correlation between WC and ba-PWV in the male group can also be explained by the fact that $\mathrm{WC}$ cannot distinguish between visceral and subcutaneous fats. ${ }^{18}$ The BRI, which is based on height and $\mathrm{WC}$, is known as a better predictor of visceral adiposity and body fat than BMI and WC. However, the predictive value of BRI for metabolic syndrome and CVD risk remains controversial. One study found its potential to identify $\mathrm{DM},{ }^{2}$ whereas another study showed that BRI is not better than classic obesity parameters in predicting metabolic syndrome. ${ }^{23}$ In our present study, BRI positively correlated with ba-PWV only in the women, which suggests that it is not a superior measurement to identify arterial stiffness in the Korean population. These discrepancies could be explained by ethnic differences, and the different characteristics and sizes of the study sample.

The ABSI, a newly proposed obesity index, was also positively associated with visceral adiposity. ${ }^{20} \mathrm{~A}$ previous study presented that ABSI could be a substantial marker of arterial stiffness in patients with type 2 DM. $^{20}$ A more recent study by Zhang et al ${ }^{18}$ indicated that waist-to-height ratio, ABSI, and BRI were related to arterial stiffness. VAI, which is based on BMI, WC, TG levels, and HDL level, is a new sexspecific indicator of fat distribution. ${ }^{9}$ In a previous study, among 5,158 community-dwelling individuals, Yang et $\mathrm{al}^{9}$ showed that VAI could be a surrogate marker for obesity assessment and its impact on atherosclerosis. Our data were consistent with those reported in the previous studies. ABSI and VAI significantly correlated with the mean ba-PWV in both the men and women in Korea. These results suggest that ABSI and VAI may be convenient and highly costeffective to apply as simple assessment tools for obesity and CVD risk in clinical practice.

This study has several strengths. To the best of our knowledge, this is the first study to examine the relationships between arterial stiffness and multiple anthropometric parameters (ABSI, BRI, and VAI) in the Korean population. Second, health behaviors such as smoking, drinking status, and physical inactivity that could influence anthropometric parameters were also assessed.

The study also has some limitations. First, it has a small sample size $(n=2,746)$. Second, it has a crosssectional design, which cannot establish a cause-effect relationship. Third, our study focuses only on the Korean population. Therefore, the results of this study cannot be applied to other regions and ethnic groups. 


\section{Conclusion}

In conclusion, among the new obesity indices, ABSI and VAI have been found to be significantly associated with arterial stiffness, as represented by the mean ba-PWV in both the men and women in Korea. Further studies with a large number of participants with various ethnicities are needed to determine the true validity of the anthropometric parameters as diagnostic indexes to identify CVD risk.

\section{Disclosure}

The authors report no conflicts of interest in this work.

\section{References}

1. Katzmarzyk PT, Janssen I, Ardern CI. Physical inactivity, excess adiposity and premature mortality. Obes Rev. 2003;4(4):257-290.

2. Chang Y, Guo X, Li T, Li S, Guo J, Sun Y. A body shape index and body roundness index: two new body indices to identify left ventricular hypertrophy among rural populations in Northeast China. Heart Lung Circ. 2016;25(4):358-364. doi:10.1016/j.hlc.2015.08.009

3. Song X, Jousilahti P, Stehouwer CD, et al. Cardiovascular and allcause mortality in relation to various anthropometric measures of obesity in Europeans. Nutr Metab Cardiovasc Dis. 2015;25(3):295304. doi:10.1016/j.numecd.2014.09.004

4. Piche ME, Poirier P, Lemieux I, Despres JP. Overview of epidemiology and contribution of obesity and body fat distribution to cardiovascular disease: an update. Prog Cardiovasc Dis. 2018;61(2):103113. doi:10.1016/j.pcad.2018.06.004

5. Ehrampoush E, Arasteh P, Homayounfar R, et al. New anthropometric indices or old ones: which is the better predictor of body fat? Diabetes Metab Syndr. 2017;11(4):257-263. doi:10.1016/j.dsx.2016.08.027

6. Afsar B, Elsurer R, Kirkpantur A. Body shape index and mortality in hemodialysis patients. Nutrition. 2013;29(10):1214-1218. doi:10. 1016/j.nut.2013.03.012

7. Moore SC. Waist versus weight: which matters more for mortality? Am J Clin Nutr. 2009;89(4):1003-1004. doi:10.3945/ajcn.2009.27598

8. Dong H, Xu Y, Zhang X, Tian S. Visceral adiposity index is strongly associated with hyperuricemia independently of metabolic health and obesity phenotypes. Sci Rep. 2017;7(1):8822. doi:10.1038/s41598-01709455-Z

9. Yang F, Wang G, Wang Z, et al. Visceral adiposity index may be a surrogate marker for the assessment of the effects of obesity on arterial stiffness. PLoS One. 2014;9(8):e104365. doi:10.1371/journal.pone. 0104365

10. Amato MC, Giordano C, Galia M, et al. Visceral adiposity index: a reliable indicator of visceral fat function associated with cardiometabolic risk. Diabetes Care. 2010;33(4):920-922. doi:10.2337/dc09-1825
11. Lee DY, Lee MY, Sung KC. Prediction of mortality with a body shape index in young asians: comparison with body mass index and waist circumference. Obesity (Silver Spring). 2018;26(6):1096-1103. doi:10.1002/oby.22193

12. Fujita M, Sato Y, Nagashima K, Takahashi S, Hata A. Predictive power of a body shape index for development of diabetes, hypertension, and dyslipidemia in Japanese adults: a retrospective cohort study. PLoS One. 2015;10(6):e0128972. doi:10.1371/journal.pone. 0128972

13. Zhao Q, Zhang K, Li Y, et al. Capacity of a body shape index and body roundness index to identify diabetes mellitus in Han Chinese people in Northeast China: a cross-sectional study. Diabet Med. 2018. doi:10.1111/dme. 13787

14. Sutton-Tyrrell K, Najjar SS, Boudreau RM, et al. Elevated aortic pulse wave velocity, a marker of arterial stiffness, predicts cardiovascular events in well-functioning older adults. Circulation. 2005;111 (25):3384-3390. doi:10.1161/CIRCULATIONAHA.104.483628

15. Tsuchikura S, Shoji T, Kimoto E, et al. Brachial-ankle pulse wave velocity as an index of central arterial stiffness. $J$ Atheroscler Thromb. 2010;17(6):658-665. doi:10.5551/jat.3616

16. Bowman SA, Clemens JC, Friday JE, Thoerig RC, Moshfeghr AJ. Food Surveys Research Group Food Patterns Equivalents Database 2011-12: Methodology and User Guide. 2014;60-61. doi:10.13140/ RG.2.1.2201.5520

17. Eckel RH, Jakicic JM, Ard JD, et al. 2013 AHA/ACC guideline on lifestyle management to reduce cardiovascular risk: a report of the American college of cardiology/American heart association task force on practice guidelines. J Am Coll Cardiol. 2014;63(25Pt B):2960-2984. doi:10.1016/j.jacc.2013.11.003

18. Zhang J, Fang L, Qiu L, Huang L, Zhu W, Yu Y. Comparison of the ability to identify arterial stiffness between two new anthropometric indices and classical obesity indices in Chinese adults. Atherosclerosis. 2017;263:263-271. doi:10.1016/j.atherosclerosis.2017.06.031

19. Yamashina A, Tomiyama H, Takeda K, et al. Validity, reproducibility, and clinical significance of noninvasive brachial-ankle pulse wave velocity measurement. Hypertens Res. 2002;25(3):359-364.

20. Bouchi R, Asakawa M, Ohara N, et al. Indirect measure of visceral adiposity 'A Body Shape Index' (ABSI) is associated with arterial stiffness in patients with type 2 diabetes. BMJ Open Diabetes Res Care. 2016;4(1):e000188. doi:10.1136/bmjdrc-2015-000188

21. Sakurai M, Miura K, Takamura T, et al. Gender differences in the association between anthropometric indices of obesity and blood pressure in Japanese. Hypertens Res. 2006;29(2):75-80. doi:10. 1291/hypres.29.75

22. Czernichow S, Bertrais S, Oppert JM, et al. Body composition and fat repartition in relation to structure and function of large arteries in middle-aged adults (the SU.VI.MAX Study). Int $J$ Obes (Lond). 2005;29(7):826-832. doi:10.1038/sj.ijo.0802986

23. Liu PJ, Ma F, Lou HP, Zhu YN. Body roundness index and body adiposity index: two new anthropometric indices to identify metabolic syndrome among Chinese postmenopausal women. Climacteric. 2016;19(5):433-439. doi:10.1080/13697137.2016.1202229

Diabetes, Metabolic Syndrome and Obesity: Targets and Therapy

\section{Dovepress}

\section{Publish your work in this journal}

Diabetes, Metabolic Syndrome and Obesity: Targets and Therapy is an international, peer-reviewed open-access journal committed to the rapid publication of the latest laboratory and clinical findings in the fields of diabetes, metabolic syndrome and obesity research. Original research, review, case reports, hypothesis formation, expert opinion and commentaries are all considered for publication. The manuscript management system is completely online and includes a very quick and fair peer-review system, which is all easy to use. Visit http://www.dovepress.com/testimonials.php to read real quotes from published authors. 